\title{
Vacancies in ordered and disordered titanium monoxide: Mechanism of $B 1$ structure stabilization
}

\author{
M.G. Kostenko a, A.V. Lukoyanov ${ }^{\text {b,c }}$, V.P. Zhukov ${ }^{\text {a }}$, A.A. Rempel ${ }^{\mathrm{a}, \mathrm{c}, *}$ \\ a Institute of Solid State Chemistry, The Ural Branch of the Russian Academy of Sciences, Pervomayskaya 91, Ekaterinburg 620990, Russia \\ ${ }^{\mathrm{b}}$ Institute of Metal Physics, The Ural Branch of the Russian Academy of Sciences, S. Kovalevskoy 18, Ekaterinburg 620990, Russia \\ ${ }^{\mathrm{c}}$ Ural Federal University named after First President of Russia B.N. Yeltsin, Mira 19, Ekaterinburg 620002, Russia
}

\section{A R T I C L E I N F O}

Article history:

Received 21 January 2013

Received in revised form

24 May 2013

Accepted 27 May 2013

Available online 31 May 2013

Keywords:

Titanium monoxide

Nonstoichiometry

Structural vacancies

Electronic structure

Ordered phase

Disordered phase

\begin{abstract}
A B S T R A C T
The electronic structure and stability of three phases of titanium monoxide $\mathrm{TiO}_{y}$ with $B 1$ type of the basic structure have been studied. Cubic phase without structural vacancies, TiO, and two phases with structural vacancies, monoclinic $\mathrm{Ti}_{5} \mathrm{O}_{5}$ and cubic disordered $\mathrm{TiO}_{1.0}$, was treated by means of firstprinciples calculations within the density functional theory with pseudo-potential approach based on the plane wave's basis. The ordered monoclinic phase $\mathrm{Ti}_{5} \mathrm{O}_{5}$ was found to be the most stable and the cubic TiO without vacancies the less stable one. The role of structural vacancies in the titanium sublattice is to decrease the Fermi energy, the role of vacancies in the oxygen sublattice is to contribute to the appearance of Ti-Ti bonding interactions through these vacancies and to reinforce the Ti-Ti interactions close to them. Listed effects are significantly pronounced if the vacancies in the titanium and oxygen sublattices are associated in the so called "vacancy channels" which determine the formation of vacancy ordered structure of monoclinic $\mathrm{Ti}_{5} \mathrm{O}_{5}$-type.
\end{abstract}

(c) 2013 Elsevier Inc. All rights reserved.

\section{Introduction}

In the majority of the binary non-stoichiometric transition metal compounds the non-stoichiometry occurs due to substitution of atoms by structural vacancies in one of the sublattices [1]. In titanium monoxide $\mathrm{TiO}_{y}$ in the both sublattices, metal and nonmetal, structural vacancies are present [2]. These vacancies can be removed only by the means of annealing at high temperature and pressure [3]. At a stoichiometric composition $(y=1)$ the fraction of vacancies is about 15 at\% in every sublattice $[2,4]$. At a nonstoichiometric composition $(y \neq 1)$ the fraction of vacant sites in the titanium sublattice is not equal to that in the oxygen sublattice. In order to demonstrate the presence of vacancies in both sublattices [5] the formula of the titanium monoxide is written as $\mathrm{Ti}_{x} \mathrm{O}_{z}$ or as $\mathrm{Ti}_{x} \mathbf{m}_{1-x} \mathrm{O}_{z} \square_{1-z}$. Here $x$ and $z$ are the fractions of occupied atomic positions in the titanium and oxygen sublattices, respectively, - and $\square$ are the symbols for structural vacancies in the titanium and in the oxygen sublattices respectively. The value $y$ in the formula $\mathrm{TiO}_{y}$ is equal to $z / x$ and may vary from 0.70 to 1.25 for disordered monoxide [2].

The structures of all the phases of titanium monoxide are derivatives of the cubic $B 1$ structure $(\mathrm{NaCl})$. Vacancies can occupy

\footnotetext{
* Corresponding author at: Institute of Solid State Chemistry, The Ural Branch of the Russian Academy of Sciences, Pervomayskaya 91, Ekaterinburg 620990, Russia. Fax: +7 3433744495 .

E-mail address: rempel@ihim.uran.ru (A.A. Rempel).
}

randomly or regularly the sites of the basic structure. Disordered phase is in thermodynamic equilibrium at temperatures higher than $1263 \mathrm{~K}$ [2]. Annealing at a temperature below $1263 \mathrm{~K}$ results in a redistribution of structural vacancies within the basic $B 1$ structure and formation of ordered phases [2,4,6,7]. Monoclinic phase $\mathrm{Ti}_{5} \mathrm{O}_{5}\left(\mathrm{Ti}_{5} \boldsymbol{\bullet}_{1} \mathrm{O}_{5} \square_{1}\right)$ appears as a result of ordering when $y=1.0[2,4,6]$. Its structure is shown in Fig. 1 . The fraction of vacant sites is $1 / 6$ in both sublattices.

All sites of the perfect $B 1$ structure sublattices are equivalent to each other. The sites of every sublattice in monoclinic structure $\mathrm{Ti}_{5} \mathrm{O}_{5}$ can be classified into four types. The criterion for this classification is the number and location of vacancies in the first, second, and third coordination spheres. The clusters for the site types are shown in Figs. 2 and 3. Each type of sites is labeled with a symbol $X_{a / b}^{c}, X$ is the symbol for chemical element in the sublattice, $a, b, c$ are the numbers of vacancies around the $\mathrm{X}$ atom in the first, second, and third coordination spheres, respectively [1]. The vacancies of every sublattice of monoclinic phase are grouped in vacancy channels orientated along the $[010]_{B 1}$ crystallographic direction [4]. Vacancies in the channel alternates by the atoms of another sublattice (Fig. 4). There are also channels of another type in the ordered structure. They are composed of vacancies of both types, metal and nonmetal. These channels are oriented along the $[112]_{B 1}$ direction and can be observed experimentally with transmission electron microscopy $[8,9]$.

There are many computational studies devoted to the analysis of vacancy formation in titanium monoxide. Goodenough [11] 


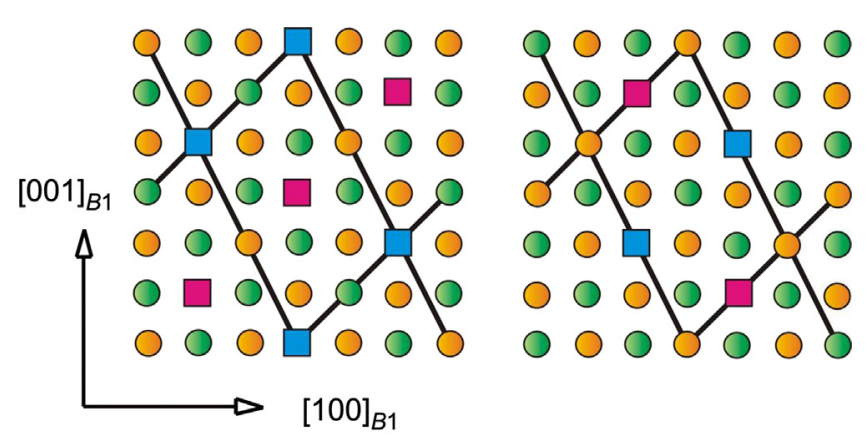

$\bigcirc$ Titanium $\quad \square$ Titanium vacancy
Oxygen $\quad \square$ Oxygen vacancy

Fig. 1. Two neighboring atomic planes perpendicular to the $[010]_{B 1}$ direction in the $\mathrm{Ti}_{5} \mathrm{O}_{5}$ structure. Unit cell boundaries are shown.
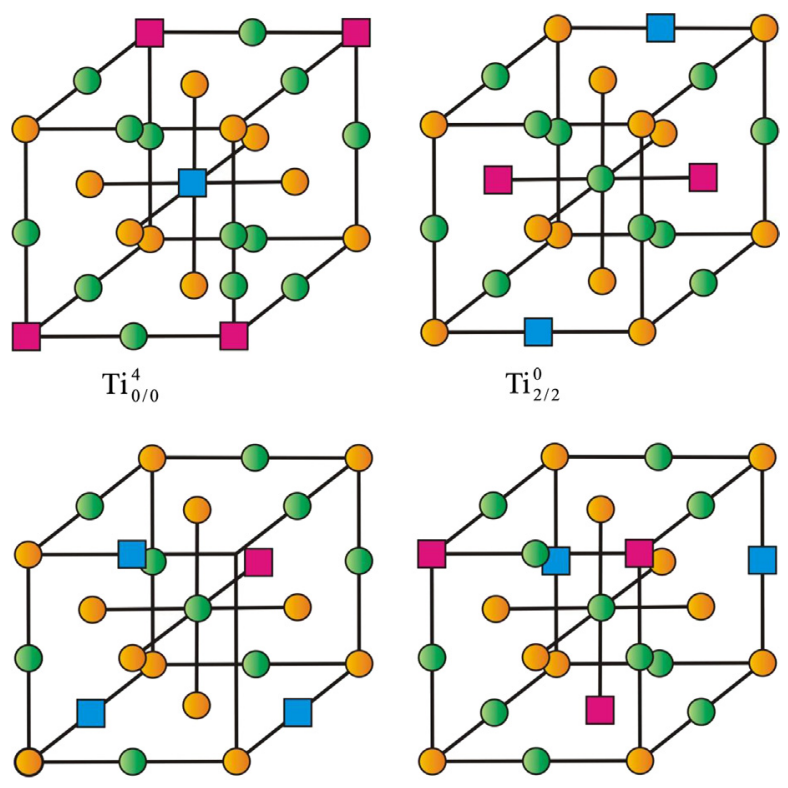

$\mathrm{Ti}_{1 / 3}^{0}$

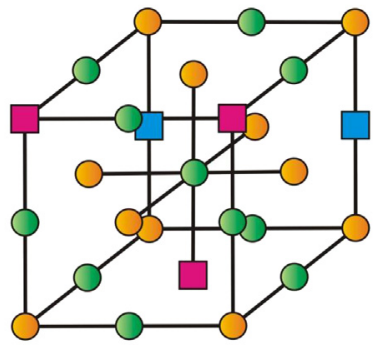

$\mathrm{Ti}_{1 / 2}^{2}$

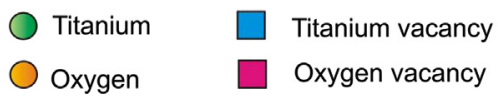

Fig. 2. Types of the titanium sublattice sites in $\mathrm{Ti}_{5} \mathrm{O}_{5}$. First and second subscripts in the symbols of types denote the number of vacancies in the first and second coordination spheres, respectively. The superscript denotes the number of vacancies in the third coordination sphere.

proposed that the vacancy creation is possible if Madelung energy loss is compensated by a reduction of the lattice constant and by an increment of the electron density in the anion holes and a depletion of the electron density in the cation holes. Huisman et al. [12] has reported that the formation of the vacancies results in shifts of the averaged band energies, changes of the band widths and position of the Fermi level, and a creation of new, so called, "vacancy states" induced by the vacancies. Gubanov et al. [13] studied the influence of the vacancies in the metallic and nonmetallic sublattices on the electronic structure employing the nonempirical Hartree-Fock-Slater method. A narrowing of the bands was found. The vacancy states are above the Fermi level and its admixture in the occupied part of the valence band is unessential. The density of electronic charge inside the nonmetal vacancies was reported to be small. This assertion contradicts to the conclusions in [11]. The metal-metal interaction through the
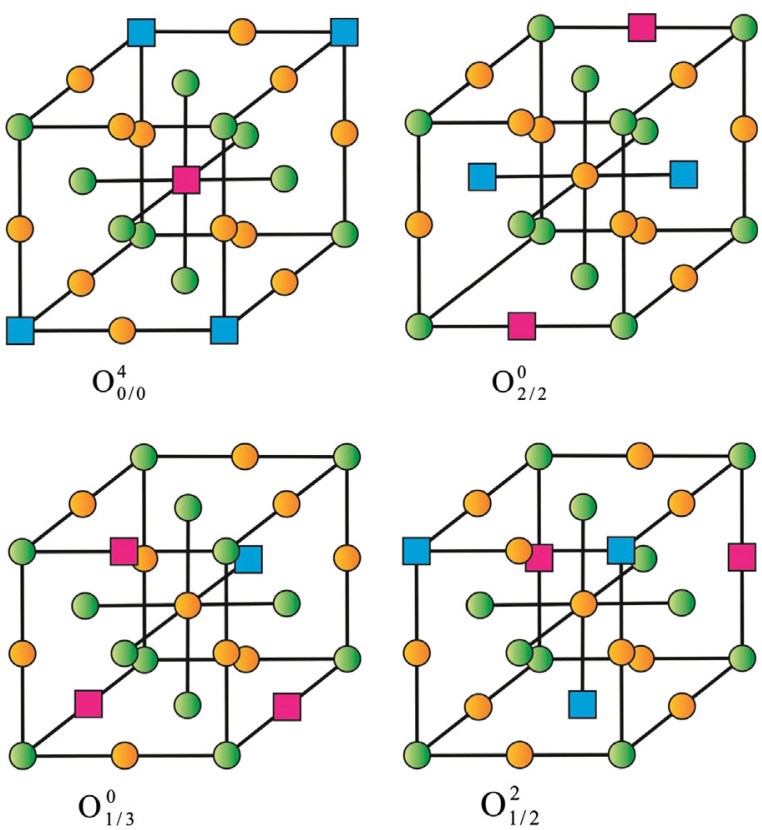

Titanium

Titanium vacancy

Oxygen vacancy

Fig. 3. Types of oxygen sublattice sites in $\mathrm{Ti}_{5} \mathrm{O}_{5}$. First and second subscripts in the symbols of types denote the number of vacancies in the first and second coordination spheres, respectively. The superscript denotes the number of vacancies in the third coordination sphere.

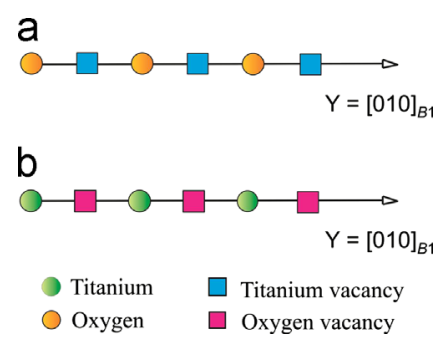

Fig. 4. Structure of vacancy channels in titanium (a) and oxygen (b) sublattices of $\mathrm{Ti}_{5} \mathrm{O}_{5}$.

oxygen vacancies was not found. On the contrary, the evidence of metal-metal interactions was reported in [14-16].

The most recent detailed computational studies of titanium monoxide were carried out in 2005. Andersson et al. [17] reported the results of LDA and GGA calculations for vacancy-free phase $\mathrm{TiO}$, monoclinic phase $\mathrm{Ti}_{5} \mathrm{O}_{5}$, and disordered phase $\mathrm{TiO}_{1.0}$. $\mathrm{TiO}$ and $\mathrm{Ti}_{5} \mathrm{O}_{5}$ were treated using the VASP code based on a plane wave basis set and projector augmented wave (PAW) potentials. $\mathrm{TiO}_{1.0}$ was treated with the Korringa-Kohn-Rostoker (KKR) Green's function technique employing multipole-corrected atomic sphere approximation. The occupied states region was found to consist mainly of $02 p$ and Ti $3 d$ states separated by a $p-d$ gap. Vacancy formation leads to the narrowing of the gap. Calculations of enthalpies of formation revealed the monoclinic phase to be preferable as compared to vacancy-free TiO. However, the use of different methods of calculation for different phases does not allow the correct comparison of full energies of these phases. Therefore the problem of stability of disordered phase was not solved.

Graciani et al. [18] studied only $\mathrm{TiO}$ and $\mathrm{Ti}_{5} \mathrm{O}_{5}$ phases that were treated with the same code as in [17]. Calculations were carried out within the GGA implementation of density functional theory proposed by Perdew et al. [19]. An analysis of partial DOS 
of $\mathrm{Ti}_{2 / 2}^{0}, \mathrm{Ti}_{1 / 3}^{0}$, and $\mathrm{Ti}_{1 / 2}^{2}$ titanium type positions revealed an enhancement of the Ti-Ti interactions through oxygen vacancy and close to the same oxygen vacancy. The hybridization between O $2 p$ and Ti $3 d$ states was not found. This means that only ionic type of chemical bond between titanium and oxygen exists. Electron density inside the oxygen vacancies was found to be higher than that inside the titanium vacancies. This factor compensates the loss in Madelung energy and contributes significantly to the stabilization of the basic $B 1$ structure.

The most detailed experimental investigation of the electronic structure of the titanium monoxide was performed by Bartkowski et al. [20]. The monoclinic phase $\mathrm{Ti}_{5} \mathrm{O}_{5}$ and the cubic phase without vacancies $\mathrm{TiO}$ were studied by X-ray and ultraviolet photoelectron spectroscopy. The experiments confirmed the presence of high density of electron states at the Fermi level and strong metallic type of chemical bond in both $\mathrm{Ti}_{5} \mathrm{O}_{5}$ and $\mathrm{TiO}$. Valence band of these phases was found to consist of $\mathrm{O} 2 p$ and $\mathrm{Ti}$ $3 d$ peaks. However, the $p-d$ gap between these peaks was not detected in contrast to the results of numerous theoretical investigations. The valence band of TiO is wider than the valence band of $\mathrm{Ti}_{5} \mathrm{O}_{5}$. No additional peaks that would be induced by vacancies in Ti $3 d$ part of valence band were found.

Although it was proposed in numerous computational studies that spontaneous formation of vacancies reduces the energy of $B 1$ lattice of titanium monoxide, there are still many problems unresolved. The numerous computational studies present contradictory results of analysis of chemical bond in the vacancy-free TiO and the ordered $\mathrm{Ti}_{5} \mathrm{O}_{5}$. The higher stability of ordered phase $\mathrm{Ti}_{5} \mathrm{O}_{5}$ as compared to the disordered phase and $\mathrm{TiO}$ is not confirmed by accurate first-principles calculations. The factors that cause the stability of disordered state are also unclear. The vacancy channels in disordered titanium monoxide are absent, but the fraction of the vacancies is about as high as in the ordered $\mathrm{Ti}_{5} \mathrm{O}_{5}$ phase. To answer all these questions, in this work first-principle calculations of the electronic structure were performed for the titanium monoxide with vacancy-free $B 1$ structure, the monoclinic phase, and the disordered cubic phase. Special attention was paid to the analysis of interaction between titanium and oxygen in different positions in $\mathrm{Ti}_{5} \mathrm{O}_{5}$ structure. In order to reveal the role of the oxygen and titanium vacancies in the disordered $\mathrm{TiO}_{y}$, calculations of the electronic structure of disordered phase with the compositions of $y=0.75$ and 1.33 were performed. These compositions correspond to the boundaries of the homogeneity region and the vacancies at least in one of the sublattices are absent.

It should be noted that at present there is a high interest to titanium monoxide as a material for cascaded oxide thermoelectric generators [21] due to its unique thermoelectric properties [22]. Additionally, titanium monoxide can be used in microelectronics as a diffusion barrier against the inter-diffusion of $\mathrm{Al}$ and $\mathrm{Si}$ $[23,24]$. Very wide homogeneity regions, high vacancy content and ordering of vacancies give an opportunity to vary the properties of the material in wide limits. Therefore, it is important to understand the influence of the composition and ordering of structural vacancies on the electronic structure of titanium monoxide.

\section{Details of the calculations}

The calculations were performed by the means of the density functional theory $[25,26]$ within the generalized gradient approximation (GGA), as implemented in the PBE [27,28] form of exchange-correlation potential. All the phases under investigation were treated with the PWSCF code of the QUANTUM-ESPRESSO package [29] based on plane waves (PW) and pseudo-potentials. The electron kinetic energy cutoff was 45 Ry. Titanium $3 s 3 p$ semi-core levels were included in the $\mathrm{Ti}$ pseudopotential.
Monkhorst-Pack scheme [30] was used for integration in the reciprocal space, the grid being $8 \times 8 \times 6$. Gaussian broadening for DOS calculations was 0.01 Ry.

To investigate the electronic structure of disordered phase, a supercell was constructed. This supercell consisted of four $\mathrm{Ti}_{5} \mathrm{O}_{5}$ unit cells and contained 96 sites of the basic $B 1$ structure. The number of atoms was 40 in every sublattice, 8 sites were vacant. There is no sufficient data about short-range order in the disordered titanium monoxide, so 80 atoms were distributed randomly on the 96 sites of the supercell. In this way, 20 super cells with different disordered distributions of atoms and vacancies were modeled. The results of calculations for them were averaged afterward with the equal weight.

The lattice constant of $\mathrm{TiO}$ with perfect $B 1$ structure was accepted to be $420.6 \mathrm{pm}$ [3]. The lattice constants of monoclinic phase are: $a=585.5, b=934.0, c=414.2 \mathrm{pm}, \beta=107^{\circ} 32$ according to the experiment [6]. These values demonstrate the tetragonal distortions along the $[100]_{B 1}$ direction of the monoclinic phase. The lattice constant of the disordered phase is $418.2 \mathrm{pm}$ [6]. The formation of vacancies results in static displacements of atoms. Therefore relaxation of atomic positions within the cell was performed for $\mathrm{Ti}_{5} \mathrm{O}_{5}$ and $\mathrm{TiO}_{1.0}$ using quasi-Newton algorithm in PWSCF code [29]. Relaxation of the cell parameters was not performed as this has a much less influence on the full energy than the introduction of vacancies and static displacements of atoms $[17,18]$.

Calculations of enthalpy of formation were carried out to estimate the role of interaction between titanium and oxygen in different phases on the stability of titanium monoxide. Entropy contribution to the energy of the phases was not taken into account as it depends on the temperature. The enthalpy of formation $\Delta H_{T_{i O} O_{y}}$ was calculated according to the following equation:

$\Delta H_{T^{2} O_{y}}=\frac{k\left(E_{\mathrm{TiO}_{y}}-N_{\mathrm{Ti}} E_{\mathrm{Ti}}-\frac{1}{2} N_{\mathrm{O}} E_{\mathrm{O}_{2}}\right)}{N}$

here $k$ is the coefficient for conversion from eV to $\mathrm{kJ} / \mathrm{mol}, E_{\mathrm{TiO}_{y}}$ is the full energy of titanium monoxide calculated for one unit cell or supercell, $N_{\mathrm{Ti}}$ and $N_{\mathrm{O}}$ are the numbers of titanium and oxygen in the cell respectively, $E_{\mathrm{Ti}}$ is the energy of titanium in its ground state calculated per one atom, $E_{\mathrm{O}_{2}}$ is the energy of an oxygen molecule that includes the effect of spin polarization, $N$ is the number of basic units ( $\mathrm{Ti}-\mathrm{O}$ atomic pairs) in the cell. To calculate $E_{\mathrm{O}_{2}}$ a primitive cubic structure was simulated. Oxygen molecules were placed on its sites. The cell parameter of the structure was accepted to be 20 times larger than the distance between oxygen atoms in the molecule to minimize the interaction between molecules.

\section{Results and discussion}

\subsection{Stability of the phases}

The Fermi energy values and enthalpies of formation calculated for the vacancy-free $\mathrm{TiO}$, the ordered monoclinic $\mathrm{Ti}_{5} \mathrm{O}_{5}$ and the disordered cubic $\mathrm{TiO}_{1.0}$ which are presented in Table 1 show that the ordered $\mathrm{Ti}_{5} \mathrm{O}_{5}$ is the most stable phase among of three. The disordered $\mathrm{TiO}_{1.0}$ is less advantageous as compared to the ordered one but the vacancy-free phase has the highest enthalpy. Formation of the vacancies in the $B 1$ structure of titanium monoxide results in a considerable decrease of the Fermi energy, the effect being larger in the case of the disordered phase.

It should be noted that the enthalpy of formation value calculated for disordered cubic phase is in excellent agreement with the experimental data [31]. Enthalpies of ordered and 
Table 1

Fermi energy $E_{F}$ and enthalpy of formation $\Delta H$ calculated in the present work for the vacancy free $\mathrm{TiO}$, monoclinic $\mathrm{Ti}_{5} \mathrm{O}_{5}$ and disordered cubic $\mathrm{TiO}_{1.0}$.

\begin{tabular}{lcc}
\hline Phase & $E_{F}(\mathrm{eV})$ & $\Delta H(\mathrm{~kJ} / \mathrm{mol})$ \\
\hline $\mathrm{TiO}$ & $18.54^{\mathrm{a}}$ & $-498.0^{\mathrm{a}}$ \\
& & $-459.2^{\mathrm{b}}$ \\
& & $-462.4^{\mathrm{c}}$ \\
$\mathrm{Ti}_{5} \mathrm{O}_{5}$ & $14.63^{\mathrm{a}}$ & $-547.6^{\mathrm{a}}$ \\
& & $-497.8^{\mathrm{b}}$ \\
& & $-507.6^{\mathrm{c}}$ \\
$\mathrm{TiO}_{1.0}$ & $14.45^{\mathrm{a}}$ & $-521.8^{\mathrm{a}}$ \\
& & $-518.4^{\mathrm{d}}$ \\
\hline
\end{tabular}

a This work, theory.

${ }^{\mathrm{b}}$ Andersson et al. [17], theory.

c Graciani et al. [18], theory.

${ }^{\mathrm{d}}$ Humprey [31], experiment.

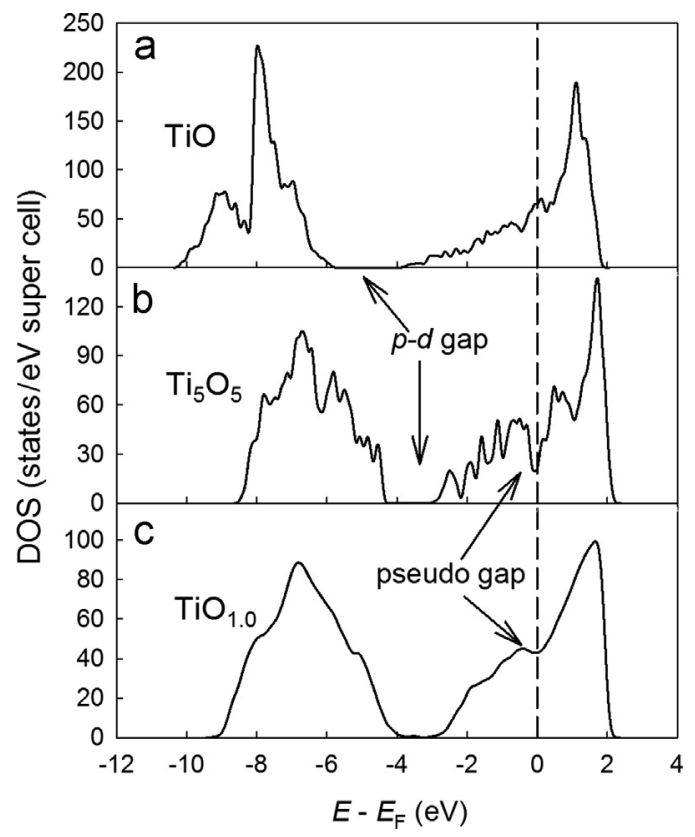

Fig. 5. Density of states for $\mathrm{TiO}(\mathrm{a}), \mathrm{Ti}_{5} \mathrm{O}_{5}$ (b) and $\mathrm{TiO}_{1.0}$ (c). All the values were calculated for the super cell with 96 sites of the basic $B 1$ structure.

vacancy-free phases are close to the values calculated in [17] and [18].

\subsection{Electronic structure}

The total densities of states calculated for the phases under investigation are shown in Fig. 5. The low energy part of the occupied states region of all three phases generally consists of $\mathrm{O}$ $2 p$. The high energy part consists of Ti $3 d$ states. TiO and $\mathrm{Ti}_{5} \mathrm{O}_{5}$ have $p-d$ gap between Ti $3 d$ and $02 p$ peaks. Disordered cubic phase has the states with non-zero density in the $p-d$ gap position. Vacancy free phase has the highest Fermi level density; monoclinic phase has the lowest one due to a pseudo-gap presence. The pseudo-gap depth decreases considerably and the Fermi level density increases under disordering of the vacancies. Appearing of the vacancies in the basic $B 1$ structure of titanium monoxide results in the narrowing of occupied states region from the bottom of the valence band to the Fermi energy, by about of $1 \mathrm{eV}$. This effect is in a good agreement with the experimental data [20].

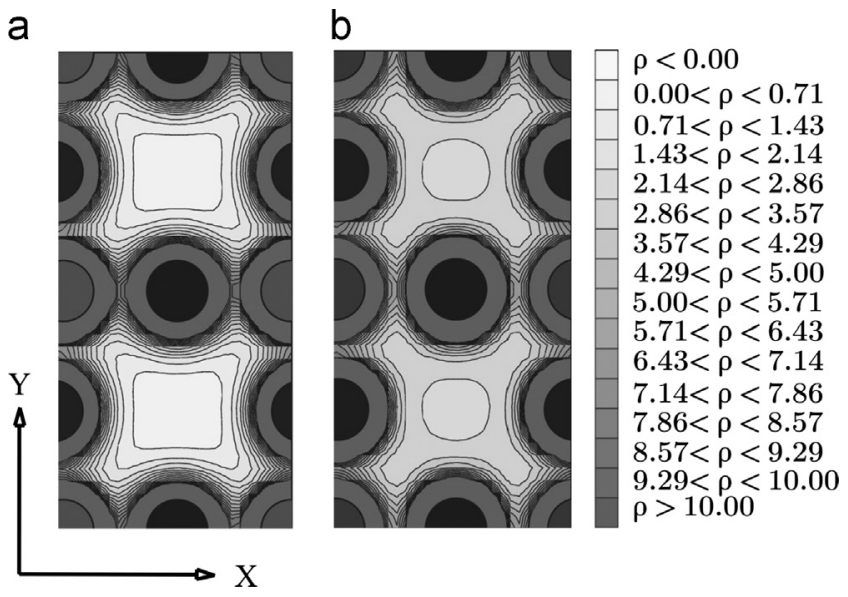

Fig. 6. Electron density map of the vacancy channels in titanium (a) and oxygen (b) sublattices. Density $\rho$ is in units $10^{-2}$ e Borh $^{-3}$.

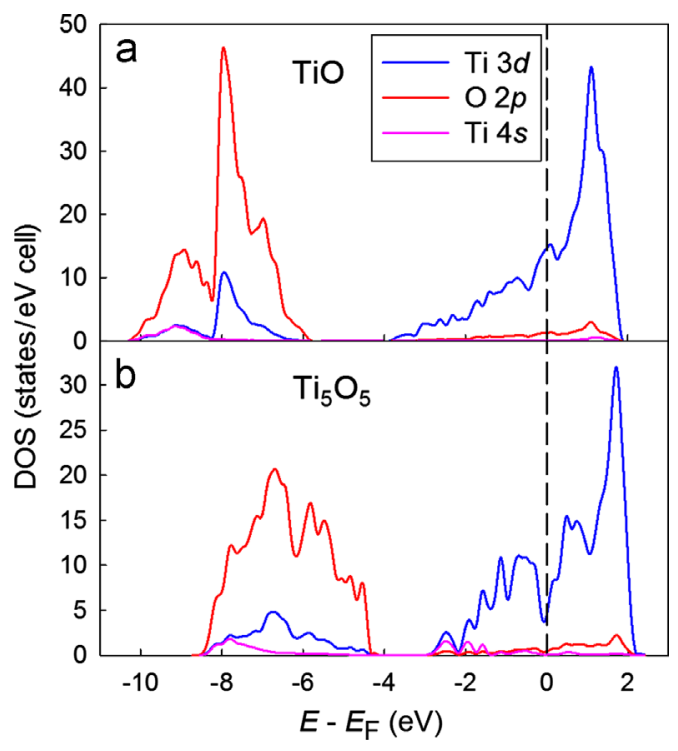

Fig. 7. Partial density of $\mathrm{Ti} 3 d$ and $4 s$ states, and $\mathrm{O} 2 p$ states for $\mathrm{TiO}$ (a) and $\mathrm{Ti}_{5} \mathrm{O}_{5}$ (b). All the values were calculated for the unit cell of the ordered phase ( 24 sites of basic $B 1$ structure).

Electron density maps of the vacancy channels in the ordered phase are presented in Fig. 6. It can be seen that the charge density in the oxygen vacancy is higher than that in the titanium vacancies. This difference compensates partially the loss in Madelung energy induced by the formation of the vacancies. In addition, accumulation of the charge in the oxygen vacancies can indicate the formation of the Ti-Ti bond interaction across these vacancies. Changes in the Ti $3 d$ states of the atoms around the oxygen vacancies lead to the redistribution of density of states near the Fermi level and the formation of the pseudo-gap. Disordered phase has no vacancy channels. Its pseudo-gap depth is very small as compared to ordered monoclinic phase. Therefore the significant change of the Ti-Ti bonding interactions requires the oxygen vacancies to be ordered.

\subsection{Mechanism of stabilization of the ordered phase}

The partial Ti $3 d$ and $\mathrm{O} 2 p$ densities of states calculated for the vacancy-free titanium monoxide and the ordered monoclinic phase are presented in Fig. 7a and b, respectively. It can be seen that the Ti $3 d$ states are present in both the high energy and the 
low energy parts of the occupied states region of $\mathrm{TiO}$ and $\mathrm{Ti}_{5} \mathrm{O}_{5}$. These states are hybridized with the $02 p$ ones in the low energy part. In [18] the Ti $3 d$ states were found to be only in the high energy part of the valence states and no indications of the $p-d$ hybridization were revealed. Analysis of the orbital configurations of the Ti $3 d$ electrons (see Fig. 8 ) indicated that some of them form the covalent bonds with $\mathrm{O} 2 p$ states in the low energy part and the others form the Ti-Ti bonds in the high energy part both in the vacancy free and the ordered monoclinic phases. The Ti-Ti interactions in the second coordination sphere cause the metallic character of titanium monoxide.

In the vacancy free $\mathrm{TiO}$ all the sites of the metallic and nonmetallic sublattices are symmetrically identical. The DOS's calculated for different configurations of the Ti $3 d$ electrons in TiO are shown in Fig. 8a. The densities of the $02 p$ configurations are presented in Fig. 9a. The $3 d_{z 2}$ and $3 d_{x 2-y 2}$ states form the Ti-O covalent bonds, the $3 d_{x y}, 3 d_{z y}$ and $3 d_{z x}$ states form the Ti-Ti bonds. The DOS's of the $3 d_{x y}, 3 d_{z y}$ and $3 d_{z x}$ states and of the $3 d_{z 2}$ and $3 d_{x 2-y 2}$ states are identical due to the symmetry of the cubic phase without vacancies.
There are three types of the atomic positions and one type of the vacancy positions in each sublattice of the monoclinic phase (see Figs. 2 and 3). The partial DOS calculated for the titanium and oxygen in different positions are presented in Figs. 8b-d and 9b-d. The $3 d_{x 2-y 2}$ states of titanium inside the vacancy channels of oxygen sublattice $\left(\mathrm{Ti}_{2 / 2}^{0}\right.$-type) are shifted from the low energy to the high energy part of the occupied states region. This effect obviously indicates the formation of interactions between atoms in $\mathrm{Ti}_{2 / 2}^{0}$ positions.

The vacancies in the ordered structure of titanium monoxide have influence not only on the state of the $\mathrm{Ti}_{2 / 2}^{0}$ atoms, but also on the titanium in the $\mathrm{Ti}_{1 / 3}^{0}$ and the $\mathrm{Ti}_{1 / 2}^{2}$ positions as well. The densities of the $3 d_{x y}, 3 d_{z y}$ and $3 d_{z x}$ states of the $\mathrm{Ti}_{1 / 3}^{0}$ and $\mathrm{Ti}_{1 / 2}^{2}$ atoms change considerably near the Fermi level and the pseudogap appears. The densities of the $\mathrm{Ti}_{1 / 3}^{0} d_{z y}$ and the $\mathrm{Ti}_{1 / 2}^{2} d_{x y}$ states decrease significantly below the Fermi level. The number of $d_{x y}$ and $d_{\mathrm{zx}}$ states of the $\mathrm{Ti}_{2 / 2}^{0}$ atoms below the Fermi level becomes larger than that of the above. All these changes indicate the enhancement of the Ti-Ti interactions formed by the $d_{x y}$ and the $d_{\mathrm{zx}}$ states of the $\mathrm{Ti}_{2 / 2}^{0}, \mathrm{Ti}_{1 / 3}^{0}$ and $\mathrm{Ti}_{1 / 2}^{2}$ atoms. On the other hand,

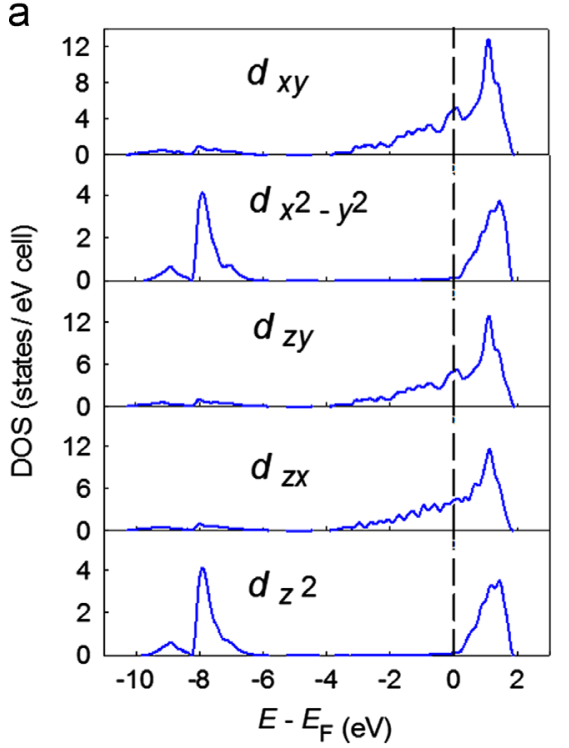

b
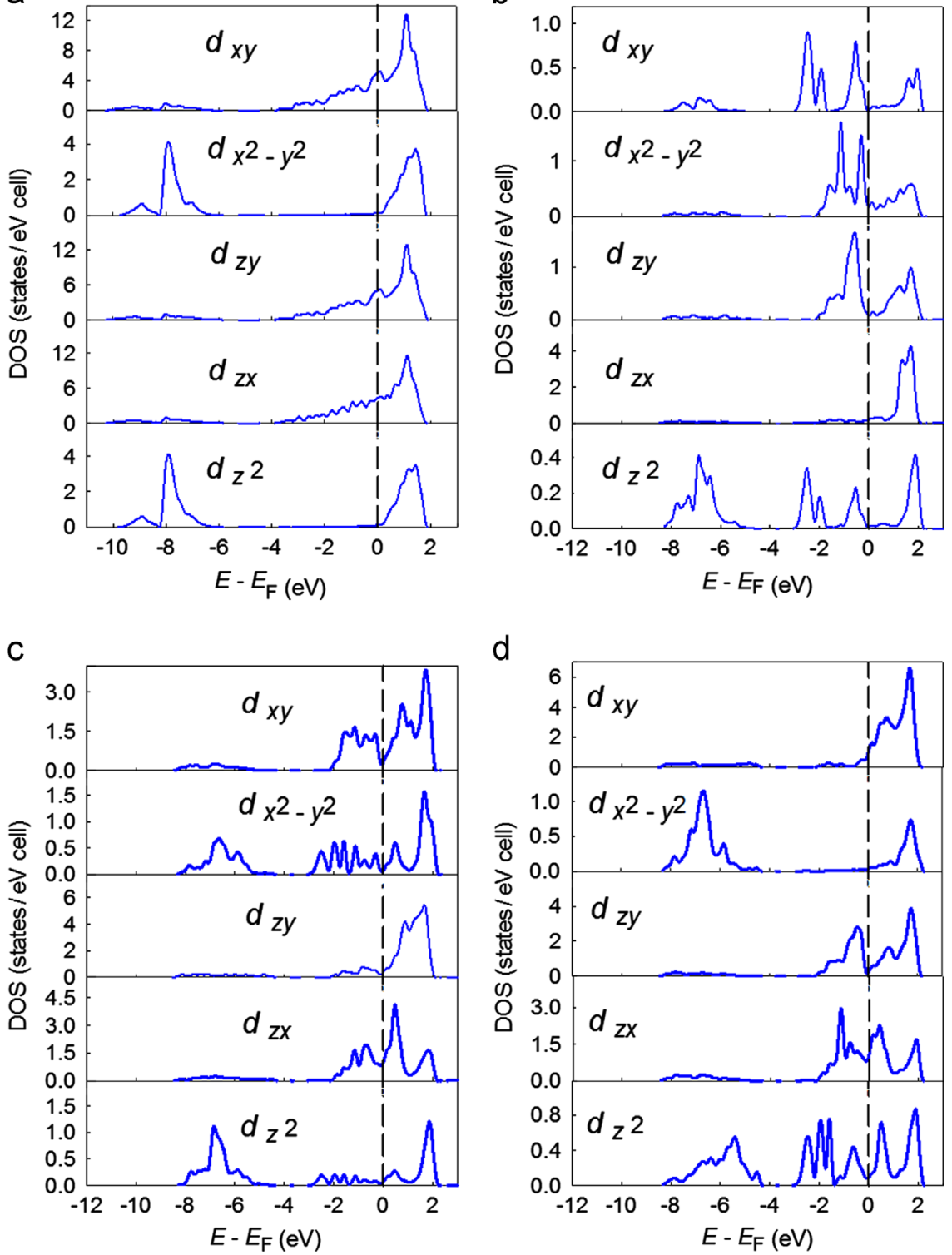

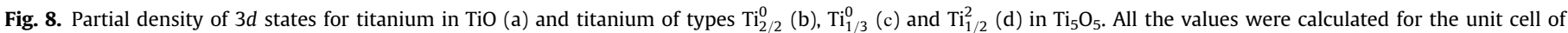
ordered phase (24 sites of basic B1 structure). 
the $02 p$ states of different atomic positions differ insignificantly. Only small destabilization of the $O p_{y}$ orbital can be noted for the atoms inside the vacancy channel of titanium sublattice. These atoms loose two of six covalent bonds with titanium. On the whole, the results of the analysis of the titanium and oxygen partial DOS depending on the types of atomic positions confirmed the conclusions of Graciani et. al. [18] with the exception of the fact of significant $3 d-2 p$ hybridization. It should be also noted that the $\mathrm{Ti} 4 s$ states which are not considered in [18] are partially hybridized with the $\mathrm{Ti} 3 d$ states both in $\mathrm{TiO}$ and $\mathrm{Ti}_{5} \mathrm{O}_{5}$. Formation of the vacancies results in changes of the densities of $\mathrm{Ti} 4 \mathrm{~s}$ states simultaneously with the changes of the Ti $3 d$ states (Fig. 7).

The specific arrangement of the oxygen and titanium vacancies is the reason for higher stability of the basic $B 1$ structure in monoclinic phase as compared to the $B 1$ structure in vacancy-free TiO. The ordering of the oxygen vacancies in the channels requires the titanium vacancies to be ordered in the same manner. At this condition all the titanium atoms take part in the stabilization mechanism described above. For instance, if the titanium vacancies are not ordered, some of the titanium atoms will be in $\mathrm{Ti}_{0 / \mathrm{b}}^{\mathrm{c}}$ positions and will have no vacancies in their first coordination spheres. Creation of titanium and oxygen vacancy channels results in the lowering of the symmetry of titanium monoxide from cubic to monoclinic one. It is also important that the most influence of the stabilization mechanism on the stability of the $B 1$ structure is
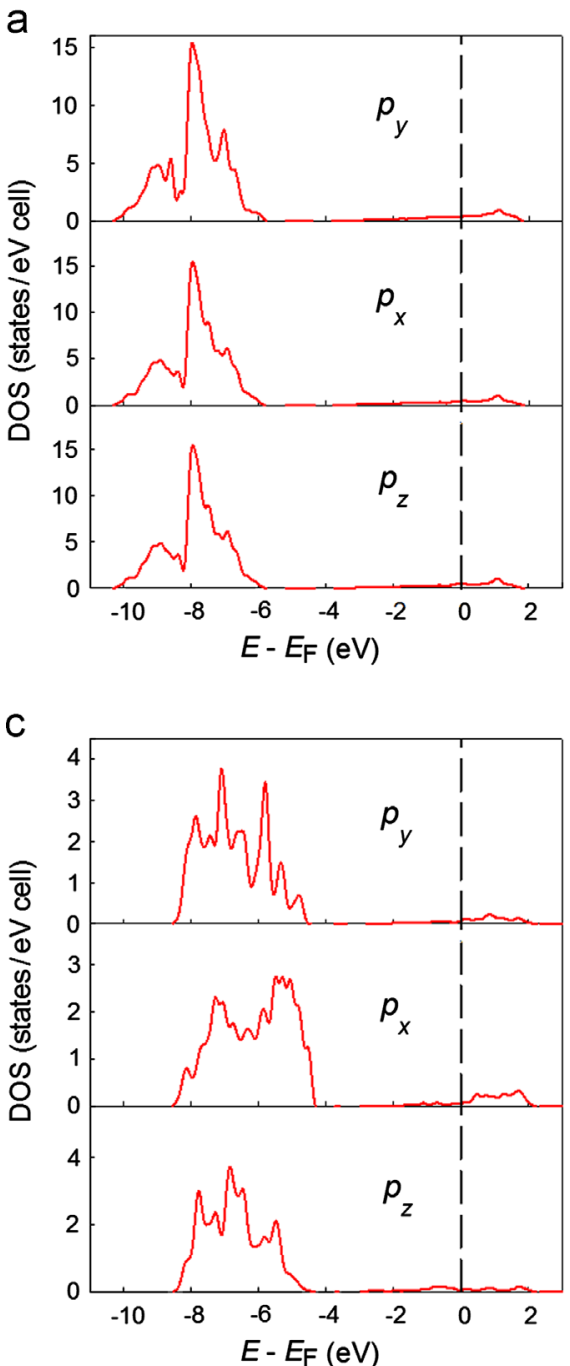

achieved at the vacancy concentration of $1 / 6$. If the vacancy concentration is less than $1 / 6$ then some Ti atoms are not affected by the oxygen vacancy channels. On the other hand, the excess of the $B 1$ lattice sites with 2 or more vacancies in the first and second coordination spheres would take place if additional vacancies were outside of the vacancy channels. This would lead to the rupture of the $\mathrm{Ti}-\mathrm{O}$ interactions in the first coordination spheres and of the Ti-Ti interactions in the second ones and, consequently, would lead to the increase of full energy of $B 1$ lattice in spite of the presence of the vacancy channels.

\subsection{Disordered phase}

Vacancy channels in the sublattices disappear and the stability of the compound decreases upon the disordering of the structural

Table 2

The lattice constant $a$, fractions of occupied positions in the titanium and oxygen sublattices $x, z$, and the Fermi energy $E_{F}$ of disordered titanium monoxide $\mathrm{TiO}_{y}$

\begin{tabular}{lllll}
\hline Composition $y$ in $\mathrm{TiO}_{y}$ & $a(\mathrm{pm})$ & $x$ & $z$ & $E_{F}(\mathrm{eV})$ \\
\hline 0.75 & 419.7 & 1 & $3 / 4$ & 16.68 \\
1.0 & 418.2 & $1 / 6$ & $1 / 6$ & 14.45 \\
1.33 & 416.0 & $3 / 4$ & 1 & 13.86 \\
\hline
\end{tabular}

b

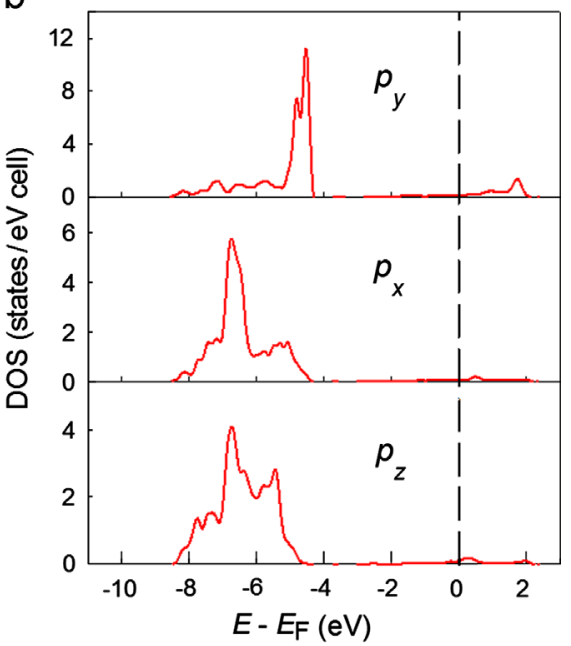

d

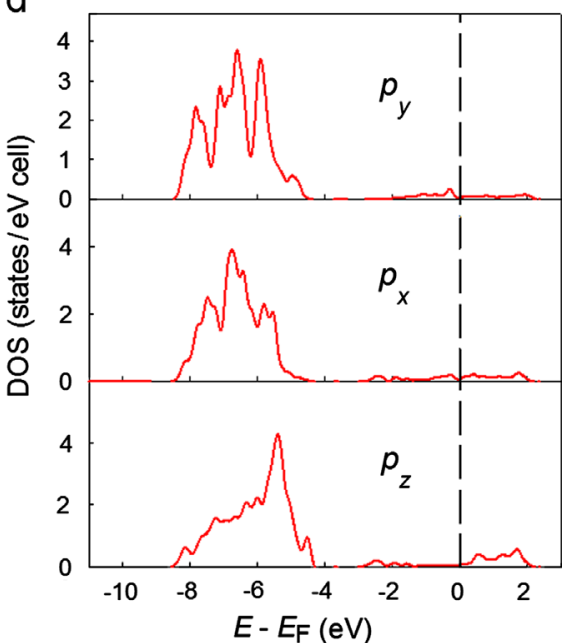

Fig. 9. Partial density of $\mathrm{O} 2 p$ states for oxygen in TiO (a) and oxygen of types $\mathrm{O}_{2 / 2}^{0}(\mathrm{~b}), \mathrm{O}_{1 / 3}^{0}$ (c) and $\mathrm{O}_{1 / 2}^{2}(\mathrm{~d})$ in $\mathrm{Ti}_{5} \mathrm{O}_{5}$. All the values were calculated for the unit cell of ordered phase (24 sites of basic $B 1$ structure). 


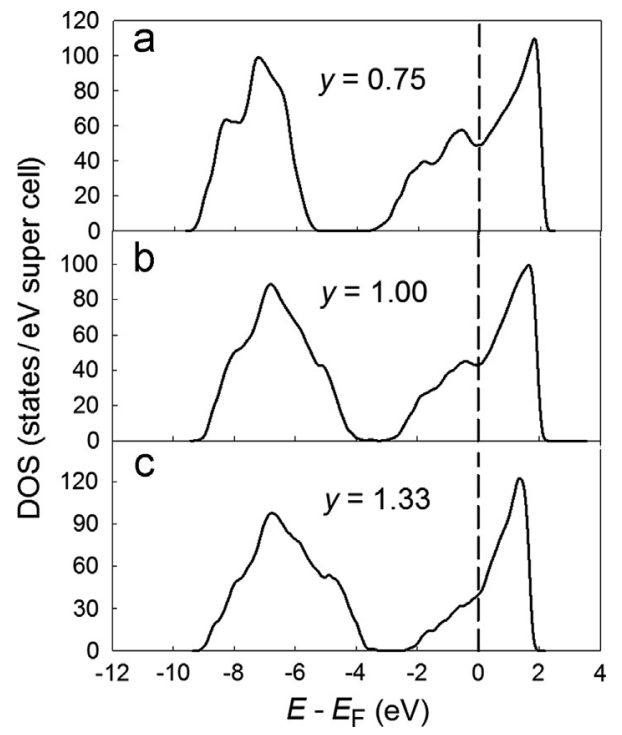

Fig. 10. Density of states calculated for supercell of disordered phase of different compositions: $\mathrm{TiO}_{0.75}$ (a); $\mathrm{TiO}_{1.0}$ (b) and $\mathrm{TiO}_{1.33}$ (c).

vacancies. The enthalpy of formation of the disordered phase is $4.7 \%$ higher than the enthalpy of the ordered phase but $4.3 \%$ less than that of the vacancy-free TiO.

The electronic structure calculations for the disordered titanium monoxide with the compositions that correspond to the homogeneity region boundaries were made to reveal the role of the vacancies in titanium and oxygen sublattices. The dependence of the vacancy concentration in the sublattices on the composition $y$ in $\mathrm{TiO}_{y}$ was studied by Valeeva et al. [5]. The dependence of the lattice constant of the disordered phase on its composition is reported in [32]. In [10] analytical dependencies were proposed for computational study. The homogeneity region boundaries of the ordered and disordered phases are not yet defined exactly enough. The ordered phase was found to be stable in the limits from $y=0.9$ to 1.1 [2] but in [33] no homogeneity region was reported. The wide homogeneity region of the disordered phase was ascertained by all the experimental investigations. Its lower boundary is in the limits from 0.7 to 0.9 and the upper boundary is in the limits from 1.25 to 1.33 [2,4-6]. In present work the homogeneity region was considered to be from 0.75 to 1.33 according to the suggestions in [10]. If $y=0.75 z$ decreases up to $3 / 4$ and $x$ increases up to 1 . On the contrary, $z$ increases up to 1 and $x$ decreases up to $3 / 4$ if $y=1.33$. Thereby, at the homogeneity region boundaries vacancy concentration is $25 \%$ for one of the sublattices and $0 \%$ for another.

The fractions of atomic positions in the sublattices and calculated Fermi energies for the disordered phase of different compositions are presented in Table 2. The corresponding electronic spectra are presented in Fig. 10. The pseudo-gap depth has the maximum when the number of oxygen vacancies is as high as possible $(y=0.75)$. If there are no vacancies on the oxygen sublattice $(y=1.33)$ no pseudo-gap can be found. On the other hand, Fermi energy decreases from 14.45 to $13.86 \mathrm{eV}$ upon the increasing of the vacancy fraction on the titanium sublattice from $1 / 6$ to $1 / 4$. Reduction of the number of the titanium vacancies up to zero results in the rise of the Fermi energy up to $16.68 \mathrm{eV}$. The Fermi energy of $\mathrm{TiO}_{0.75}\left(\mathrm{Ti}_{1} \mathrm{O}_{3 / 4}\right)$ is $10 \%$ less than that of $\mathrm{TiO}$. In the case of $\mathrm{TiO}_{1.33}\left(\mathrm{Ti}_{3 / 4} \mathrm{O}_{1}\right)$ this difference increases up to $25 \%$. Thereby, vacancies in the titanium sublattice affect Fermi energy much more than vacancies in the oxygen sublattice.

\section{Conclusion}

The first-principles calculations of the electronic structure and enthalpy of formation realized for the vacancy-free titanium monoxide $\mathrm{TiO}$, the ordered phase $\mathrm{Ti}_{5} \mathrm{O}_{5}$, and the disordered phase $\mathrm{TiO}_{y}$ demonstrated that the vacancy-free $\mathrm{TiO}$ is the less stable phase and ordered $\mathrm{Ti}_{5} \mathrm{O}_{5}$ is the most stable one. The results of calculations are in agreement with the experimental data and the results of the other known computational studies. The detailed analysis of the electronic structures revealed that the formation of the titanium vacancies decreases the Fermi energy significantly. It is the main factor for stabilization of the basic $B 1$ structure of both ordered and disordered phases of titanium monoxide. The role of the oxygen vacancy was found to be mainly in the reinforcement of the $\mathrm{Ti}-\mathrm{Ti}$ bond interactions through and close to the vacancy. This effect causes the ordering of vacancies in the sublattices and therefore the formation of the ordered monoclinic phases $\mathrm{Ti}_{5} \mathrm{O}_{5}$ that is more stable than the disordered one. The analysis of the $3 d$ states of titanium in different positions in $\mathrm{Ti}_{5} \mathrm{O}_{5}$ structure suggests that the ordering on both sublattices should occur in the same way.

\section{Acknowledgments}

Financial support by the Russian Foundation for Basic Research (Grant nos. 10-03-00164 and 10-02-00546a), by the Ural Brunch of the Russian Academy of Sciences (Project nos. 12-M-23-2001 and 13-3-NP-532), and by the Grant MK-3376.2011.2 is gratefully acknowledged. All the calculations were performed using «Uran» supercomputer of IMM UB RAS.

\section{References}

[1] A.I. Gusev, A.A. Rempel, A. Magerl, Disorder and order in strongly nonstoichiometric compounds, Transition Metal Carbides, Nitrides and Oxides, Springer, Berlin, 2001.

[2] D. Watanabe, et al., The Chemistry of Extended Defects in Non-Metallic Solids, in: L. Eyring, M. O'Keefe (Eds.), North-Holland, Amsterdam, 1970, p. 238

[3] A. Taylor, N.J Doyle, The Chemistry of Extended Defects in Non-Metallic Solids, in: L. Eyring, M. O'Keefe (Eds.), North-Holland, Amsterdam, 1970, p. 523.

[4] A.A. Valeeva, et al., Inorg. Mater. 37 (2001) 603.

[5] A.A. Valeeva, et al., Phys. Rev. B 75 (2007) 094107-1.

[6] D. Watanabe, et al., Acta Crystallogr. 23 (1967) 307.

[7] D. Watanabe, et al., J. Phys. Soc. Jpn. 25 (1968) 292.

[8] A.A. Valeeva, et al., JETP Lett. 77 (2003) 25.

[9] A.A. Valeeva, et al., Phys. Solid State 45 (2003) 87.

[10] M.G. Kostenko, A.A. Rempel, Phys. Solid State 53 (2011) 1909

[11] J.B. Goodenough, Phys. Rev. B 5 (1972) 2764.

[12] L.M. Huisman, et al., Phys. Rev. B 22 (1980) 991.

[13] V.A. Gubanov, et al., J. Phys. Chem. Solids 46 (1985) 383.

[14] G. Hörmandinger, et al., Solid State Commun. 68 (1988) 467.

[15] F. Schlapansky, et al., Z. Phys. B 75 (1989) 187.

[16] S.R. Barman, D.D. Sarma, Phys. Rev. B 49 (1994) 16141.

[17] D.A. Andersson, et al., Phys. Rev. B 71 (2005) 144101-1.

[18] J. Graciani, et al., Phys. Rev. B 72 (2005) 054117.

[19] J.P. Perdew, et al., Phys. Rev. B 46 (1992) 6671.

[20] S. Bartkowski, et al., Phys. Rev. B 56 (1997) 10656.

[21] L. Zhang, et al., Mater. Trans. 49 (7) (2008) 1675.

[22] N. Okinaka, T. Akiyama, ISIJ Int. 50 (2010) 1296.

[23] K.G. Grigorov, et al., Vacuum 51 (1998) 153.

[24] I.N. Martev, Vacuum 58 (2000) 327.

[25] W. Kohn, L.J. Sham, Phys. Rev. 140 (1965) A1133.

[26] R.O. Jones, O. Gunnarsson, Rev. Mod. Phys. 61 (1989) 689.

[27] J.P. Perdew, et al., Phys. Rev. Lett. 77 (1996) 3865.

[28] Pseudopotentials, Ti.pbe-sp-van_ak.UPF and O.pbe-rrkjus.UPF from the 〈www.quantum-espresso.org distribution 〉.

[29] P. GiannozziS, et al., J. Phys. Condens. Matter 21 (2009) 395502.

[30] H.J. Monkhorst, J.D. Pack, Phys. Rev. B 13 (1972) 5188.

[31] G.L. Humprey, J. Am. Chem. Soc. 73 (1951) 1587.

[32] A.I. Gusev, A.A. Valeeva, Phys. Solid State 45 (2003) 1242.

[33] J.L. Murray, H.A. Wriedt, Bull. Alloy Phase Diagrams 8 (1987) 148. 